\title{
Renal infarction as an uncommon cause of abdominal pain.
}

\section{A case report}

\author{
Lucio Brugioni, ${ }^{1}$ Chiara Gozzi, ${ }^{1}$ Pietro Martella, ${ }^{1}$ Andrea Borsatti, ${ }^{1}$ Francesca De Niederhausern, ${ }^{1}$ \\ Elisa Romagnoli, ${ }^{1}$ Dimitriy Arioli, ${ }^{1}$ Jacopo Catellani, ${ }^{2}$ Fabio Brugioni ${ }^{2}$ \\ ${ }^{1}$ Internal Medicine, Critical Area Unit, Policlinico Modena; ${ }^{2}$ Emergency Medicine, University of Modena and Reggio Emilia, \\ Italy
}

\begin{abstract}
Renal infarction is a rare cause of abdominal pain whose diagnosis is often misunderstood or severely delayed. The difficulty in identifying this time-dependent condition greatly limits the possibilities of therapeutic intervention and determines the loss of renal parenchyma that could have been saved with prompt diagnosis. It is, therefore, essential to include renal infarction in the differential diagnosis in case of abdominal pain and to identify this pathology beforehand. We present a case of a 65 -yearold male with atrial fibrillation in therapy with Edoxaban who was admitted to the hospital for acute onset of widespread abdominal pain with nausea, vomit, and a worsening of renal function according to the laboratory tests. An abdominal computed tomography with contrast confirmed the presence of a bilateral renal infarction. The patient developed chronic kidney disease and was discharged on anticoagulant therapy. The aim of this paper is, therefore, to increase physician awareness towards this condition, the best opportunity to diagnose early renal infarction and to establish acute and long-term therapy.
\end{abstract}

\section{Case Report}

A 65-year-old Ghanaian man came to the emergency department of our hospital complaining of abdominal pain that suddenly arose two days

Correspondence: Lucio Brugioni, Internal Medicine, Critical Area Unit, Policlinico Modena, via del Pozzo 71, 41121 Modena, Italy.

Tel.: +39.059.422549.

E-mail: brugioni.lucio@aou.mo.it

Key words: Renal infarction; abdominal pain; acute renal injury; anticoagulation.

Contributions: all authors contributed equally to the writing of the article.

Conflict of interests: the authors declare no potential conflict of interests.

Ethics approval: not required.

Informed consent: the patient gave informed consent.

Received for publication: 10 April 2020.

Revision received: 4 May 2020.

Accepted for publication: 4 May 2020.

This work is licensed under a Creative Commons Attribution NonCommercial 4.0 License (CC BY-NC 4.0).

${ }^{\circ}$ Copyright: the Author(s), 2020

Licensee PAGEPress, Italy

Italian Journal of Medicine 2020; 14:172-175

doi:10.4081/itjm.2020.1286 earlier associated with nausea and vomiting. His past medical history included hypertensive cardiopathy with atrial fibrillation on Edoxaban $60 \mathrm{mg}$ therapy $\left(\mathrm{CHA}_{2} \mathrm{DS}_{2}\right.$-VASc Score 2), ethyl abuse, and idiopathic eyelid ptosis in the neurological follow-up. At home, the patient also took amlodipine and bisoprolol. General conditions were careless, and the patient reported not to regularly comply with the prescribed pharmacological therapy. At the check-up, he was apyretic, blood pressure 160/85 $\mathrm{mmHg}$, heart rate 65 beats per minute, $97 \%$ oxygen saturation; upon inspection, the patient appeared significantly dehydrated with dry skin and mucous membranes. He complained of an important diffuse abdominal tenderness with an initial defense reaction in the hypogastrium and on the right side. The point of care ultrasound revealed the absence of free liquid in the abdomen, kidneys in place without signs of hydronephrosis, inferior vena cava completely collapsing on the inspiration. Laboratory tests showed elevation of $\mathrm{C}$ reactive protein $(\mathrm{CRP})(2.4 \mathrm{mg} / \mathrm{dL}$, reference $<0.5)$, d-Dimer $(3740 \mathrm{ng} / \mathrm{mL}$ reference $<500)$, creatinine $(2.07 \mathrm{mg} / \mathrm{dL}$, baseline $1.2 \mathrm{mg} / \mathrm{dL})$, sodium $143 \mathrm{mEq} / \mathrm{L}$ (reference $135-145 \mathrm{mEq} / \mathrm{L}$ ), blood urea nitrogen $43 \mathrm{mg} / \mathrm{dL}$ (reference 15-55 $\mathrm{mg} / \mathrm{dL}$ ), presence of proteinuria and microhematuria on the physical-chemical urine analysis. A complete ultrasound of the abdomen was performed, which did not provide further relief elements. The patient was hospitalized at our operating unit where: in the suspicion of a prerenal origin of the acute kidney injury, intravenous hydration with crystalloid fluid was started $(80 \mathrm{~mL} / \mathrm{h})$, and we decided to stop the 
administration of Edoxaban in favor of calcium heparin (12,500 IU bid, a patient of 75 kilos). The blood control tests performed on the second day of hospitalization showed a stable compromised renal function, increased CRP $(33.2 \mathrm{mg} / \mathrm{dL})$, leukocytosis, increased lactate dehydrogenase (LDH) $(2404 \mathrm{U} / \mathrm{L}$, reference 230-460). The patient was apyretic, and diuresis was regular, but abdominal pain was still present and poorly controlled by the administration of paracetamol; an episode of macrohematuria also occurred. It was, therefore, decided to carry out a new ultrasound evaluation of the abdomen, that detected a hypoechoic area with a three-angular aspect at the spleen level and a hyperechogenicity of the medullary pyramids of the right kidney associated with a reduction of the echo-color Doppler signal into the cortical area. In consideration of these suggestive findings, highly suspected for multiple cardioembolic ischemic lesions, an enhanced-computed tomography (CT) of the abdomen was performed. The exam revealed a bilateral renal infarction with greater involvement of the right kidney and a smaller ischemic lesion in the spleen. In particular, the right kidney was completely devoid of contrast impregnation as from occlusion of the renal artery; only a small lower polar portion was vascularized by an accessory renal artery (Figure 1). The left kidney was only marginally affected by some ischemic lesions likely due to sub-segmental occlusions. The ischemic wedge shape lesion of the posterior portion of the spleen was also documented (Figure 2).

Since ischemic lesions have been present for over three days based on what the patient referred to about the onset of the pain, we decided not to perform any fibrinolytic procedures and to continue the anticoagulant without anti-aggregation therapy, attributing the cause of the embolization process to inadequately anticoagulated atrial fibrillation. For these reasons, no further investigations (except transthoracic echocardiogram that was negative for cardiac thrombi) were carried out to exclude other conditions conferring high thrombotic risk such as cancer - there were no alarm symptoms - or antiphospholipid antibodies.

The patient did not experience further episodes of macrohematuria, and subsequent blood tests showed a progressive reduction in CRP and LDH values. Despite the disappearance of abdominal pain and the improvement of laboratory tests, renal function remained stably reduced; the creatinine clearance (Cockcroft-Gault equation) performed on the seventh day was $34 \mathrm{ml} / \mathrm{min}$. The patient was discharged resuming Edoxaban (30 $\mathrm{mg}$ once daily) and recommending greater adherence to therapy; blood tests performed fifteen days after discharge showed normalization of inflammation indices but persistent chronic kidney disease (stage 3b National Kidney Foundation).

\section{Discussion}

Renal infarction is a rare pathology: in an extensive study of autopsies, the incidence was $1.4 \%$, in patients with atrial fibrillation is around $2 \%$, but the frequency is probably higher since the diagnosis is frequently missed. The major causes of renal infarction are cardioembolic diseases (atrial fibrillation, cardiomyopathy, artificial valve, endocarditis, thrombi from the suprarenal aorta or left ventricle); renal artery injury (dissection, trauma, Marfan syndrome, Polyarteritis nodosa, compression) and hypercoagulable states

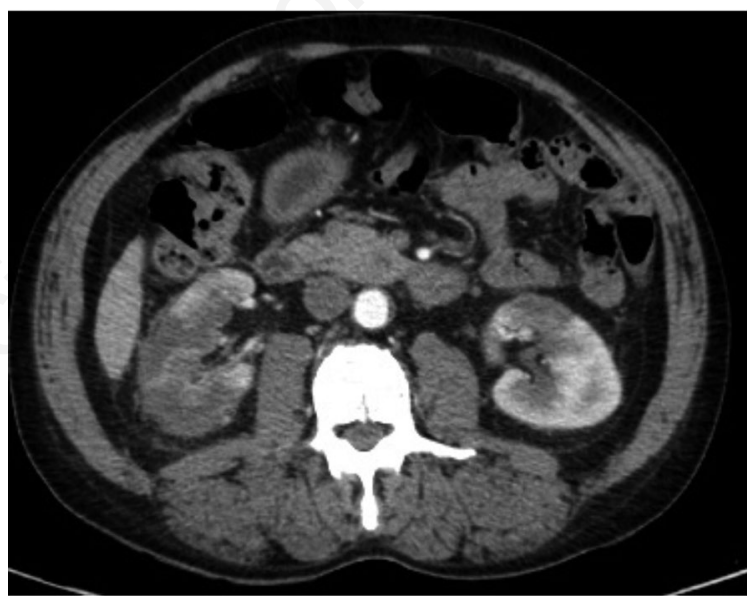

Figure 1. Contrast-enhanced computed tomography with evidence of hypodense and vascular areas affecting the kidneys bilaterally, with more significant savings of the left kidney.

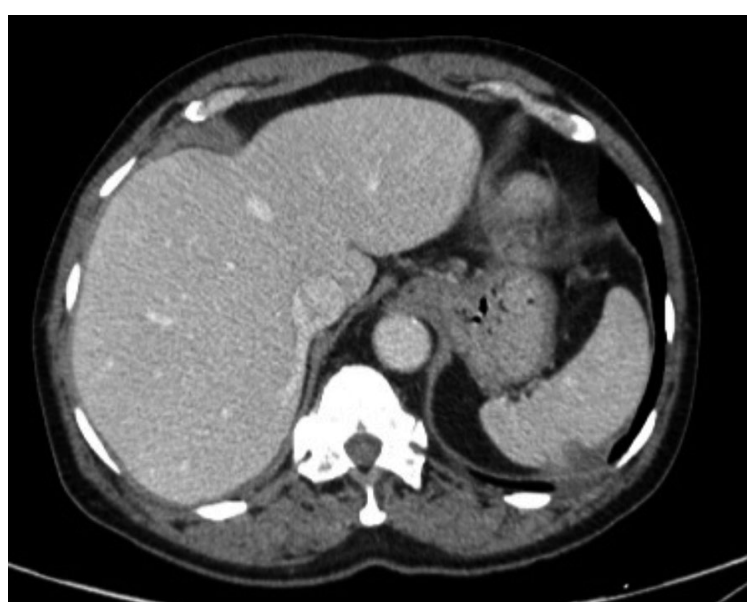

Figure 2. Ischemic wedge-shaped lesion of the spleen. 
(malignancy, antiphospholipid antibody syndrome, paroxysmal nocturnal hemoglobinuria). ${ }^{1-3}$

Renal infarction, due to its rarity and the presentation modality that often mimics other pathologies (nephrolithiasis and pyelonephritis), severely tests the clinician's diagnostic skills. It is also a time-dependent pathology whose early identification is essential to be able to carry out effective therapy in preserving the greatest portion of renal parenchyma as possible. It is therefore essential that this condition becomes part of the differential diagnosis process when the patient accesses the Emergency Department complaining of abdominal pain, in particular some clinical and laboratory characteristics that have been most frequently described, should lead the clinician to suspect a renal infarction. First of all, the presence of a real thromboembolic risk: in our case, the patient was affected by a chronic atrial fibrillation and was on Edoxaban therapy, but anamnesis uncovered an irregular assumption, so a condition of high thrombotic risk could be delineated. Secondly, many authors have underlined that the clinical manifestations of renal infarction are varied; however, it is frequently mistaken for renal colic or pyelonephritis. Unlike renal colic, renal infarction is refractory to therapy with nonsteroidal anti-inflammatory drugs (NSAIDs); compared to nephrolithiasis, ultrasound signs, shaking shiver at the onset and presence of bacteriuria are absent. Macrohematuria and hypertension secondary to the release of high quantities of renin may be present but have a relatively low frequency as well as the presence of ischemic lesions affecting other organs, which, although strongly indicative of a thromboembolic background disease, are only rarely detected. From a laboratory point of view, in addition to proteinuria and microhematuria, in case of renal infarction, strong phlogistic activation and high DDimer value are present, but the most indicative laboratory examination of is LDH level, which showed to be closely correlated with kidney infarction. Although this index is very susceptible, it is at the same time non-specific; therefore, the ideal would be to dose urinary LDH, whose elevation is highly suspect for renal infarction. Creatinine values may remain within normal limits or may be increased based on the portion of renal parenchyma affected by ischemia, the ability of the contralateral kidney to vicariate and renal function before the ischemic event; obviously in circumstances of bilateral lesions or patients with solitary functioning kidney, an acute injury will be observed. ${ }^{4,5}$ In the case described the patient experienced acute kidney injury due to bilateral involvement with almost complete exclusion from the bloodstream of the right kidney, increase in CRP, DDimer, and LDH, and the presence of proteinuria and macrohematuria was observed.
In the presence of a patient with renal colic-like abdominal pain at high thrombotic risk, the ineffectiveness of therapy with NSAIDs in the management of pain, no evidence of ultrasound signs of hydroureteronephrosis, an increase in D-Dimer and $\mathrm{LDH}$, and the presence of proteinuria and microhematuria, it is fundamental to take into consideration the hypothesis of renal infarction. About the diagnostic pathway, radiological diagnosis can be made with contrast-enhanced CT scan, magnetic resonance imaging, or renal scintigraphy. The last one besides providing indications on the vascularization of the kidney it also provides information on the residual renal function which is useful in order to plan a fibrinolytic rescue treatment; it is therefore highly sensitive and specific but challenging to perform in urgent conditions, as well as Magnetic Resonance Imaging. Ultrasound is not very sensitive in identifying this condition and is operator dependent, therefore, it has a marginal role in the diagnosis of renal infarction. Hence, we suggest in patients with the above criteria, first to perform an abdomen CT scan in order to exclude the presence of renal lithiasis not visible on ultrasound and possibly responsible for abdominal pain; in the event of a failure to document a kidney stone the investigation must be completed with a contrast-enhanced CT scan. ${ }^{6}$

Concerning the treatment, despite the weak evidence due to the lack of randomized controlled studies, intra-arterial thrombolytic and percutaneous endovascular therapies, anticoagulants, and antiplatelet therapy can be undertaken. The choice will be influenced by the type of lesion, by its dating, the presence of impaired renal function, and the appearance of complications such as hypertension refractory to therapy or macrohematuria. New lesions, possibly within twenty-four hours, mainly if they affect the renal artery or its segmental branches or associated with acute renal failure or in a patient with solitary functioning kidney, should be evaluated for endovascular thrombolytic procedures.

Furthermore, partial occlusions have also been observed to have a better outcome after reperfusion therapy.

Patients who underwent angioplasty or stent placement should be treated with aspirin and clopidogrel for three to six months after the procedure followed by aspirin alone after that; patients with a stent placed and atrial fibrillation should be treated with anticoagulation and low-dose aspirin.

Symptomatic patients with imaging that suggesting recent infarction but unlikely to benefit from revascularization must be anticoagulated at least for six months; the duration of anticoagulation depends on the presence of permanents risk factors as atrial fibrillation. 
Asymptomatic patients with remote infarct can be managed with aspirin alone if other indications for anticoagulation are absent. ${ }^{7}$

In our case, we decided not to perform fibrinolytic therapy due to the onset of symptoms for more than three days at the time of diagnosis, with therefore an almost complete loss of the functionality of the renal parenchyma involved. As a consequence of prolonged ischemia, despite optimizing anticoagulant therapy, no improvement in renal function was observed. In our poorly compliant patient, we prescribed the most comfortable therapy resuming Edoxaban after adequate counseling, because-as well known- this class of drugs requires vigilance, high compliance level to the treatment and regular follow-up.

\section{Conclusions}

Kidney infarction is a rare but also underestimated condition: the symptoms often are nonspecific and mimic more frequent pathologies such as nephrolithiasis and pyelonephritis, thus leading to a diagnostic delay or failure. This leads to the lack of possibility of carrying out an early treatment with the aim of preserving as much of the renal parenchyma as possible, improving the patient's prognosis, especially in terms of complications and residual renal function.
Moreover, the lack of identification of the cause of renal infarction exposes the patient to further episodes and possibly to the involvement of other organs with also increased mortality.

\section{References}

1. Hazanov N, Somin M, Attali M, et al. Acute renal embolism. Forty-four cases of renal infarction in patients with atrial fibrillation. Medicine (Baltimore) 2004;83:292-9.

2. Antopolsky M, Simanovsky N, Stalnikowicz R, et al. Renal infarction in the ED: 10-years experience and review of the literature. Am J Emerg Med 2012;30:1055-60.

3. Lessman RK, Johnson SF, Coburn JW, et al. Renal artery embolism: clinical features and long-term followup of 17 cases. Ann Intern Med 1978;89:477-82.

4. Huang CC, Lo HC, Huang HH, et al. ED presentations of acute renal infarction. Am J Emerg Med 2007;25: 164-9.

5. Korzets Z, Plotkin E, Bernheim J, et al. The clinical spectrum of acute renal infarction. Isr Med Assoc J 2002;4:781-4.

6. Suzer O, Shirkhoda A, Jafri SZ, et al. CT features of renal infarction. Eur J Radiol 2002;44:59-64.

7. Silverberg D, Menes T, Rimon U, et al. Acute renal artery occlusion: Presentation, treatment, and outcome. J Vasc Surg 2016;64:1026. 